\title{
LEOPARDI NELLE RIVISTE ITALIANE TRA LE DUE GUERRE
}

Daniel RAFFINI

Sapienza Università di Roma

\begin{abstract}
En): The aim of this essay is to highlight some moments of Leopardi's reception in the Italian magazines of the period between the two World Wars, the starting point for the reception of the poet during the twentieth century. Starting from the central role played by the magazine «La Ronda», the essay will move on through the analysis of Leopardian reception in magazines whose contribution has been less studied, such as «La Fiera Letteraria», «Il Selvaggio» e «Il Frontespizio». Each magazine tried to link Leopardian poetic to his own artistic and political ideal. Beyond ideological intentions, Leopardi's reception in the 1920s and 1930s shows the rediscovery of minor works and prose and the willingness to insert the poet into the canon of Italian literature.
\end{abstract}

Keywords (En): Leopardi; reception; magazines; 900

Parole chiave (It): Leopardi; ricezione; riviste; 900

A partire dagli anni Sessanta del XX secolo si assiste a un forte incremento di studi sulla ricezione di Leopardi. In questa epoca i critici e gli storici della letteratura iniziano a prendere in esame come il Novecento aveva interpretato o stava interpretando il poeta. Anche se la maggior parte degli osservatori è concorde nell'individuare l'inizio della ricezione novecentesca leopardiana con «La Ronda», l'approccio della rivista viene spesso criticato. «La Ronda» è accusata di aver dato una visione di Leopardi riduttiva, strumentale e fuorviante. Questi critici, colti dallo zelo di restituire al poeta la dignità toltagli, cedono all'atto paternalistico di voler imporre il proprio punto di vista come il migliore possibile, ponendosi così al di fuori della storia. Visti oggi, gli anni Sessanta risultano invece quanto mai immersi nel flusso della storia. I limiti della ricezione novecentesca di Leopardi vengono individuati per primo da Massimiliano BONI, che nel 1967 pubblica il saggio Leopardi e La Ronda, in cui scrive:

\footnotetext{
Ora anche la pagina leopardiana de la Ronda [...] viene considerato marginale avvenimento senza alcuna importanza nella storia della fortuna del Leopardi, anzi, peggio, un pretesto per la difesa di idee che nulla avevano a che fare con il Leopardi e quindi un tradimento alla sua vera ed effettiva lezione. (1967:9-10)
}

Tale visione risulta inficiata dal pregiudizio verso la rivista, catalogata sbrigativamente come organo ufficiale del ritorno all'ordine e in quanto tale sospettata di filofascismo ante litteram. Nei passi dei critici citati da Boni, i detrattori de «La Ronda» si pongono il problema della giusta interpretazione di Leopardi. Carlo Bo ad esempio sostiene che quello de «La Ronda» è un Leopardi imperdonabilmente snaturato; ma sembra che Bo tenda a idealizzare il poeta, vedendo in lui uno spirito più ribelle di quanto non fosse. Di contro BONI sostiene che «Leopardi, sotto l'aspetto letterario [...] è un uomo d'ordine» (1967: 14), 
seppur capace di contemplare il disordine. D'altro canto, secondo BONI i rondisti sapevano che «l'eleganza di Leopardi [...] non ha nulla da vedere colla purità, proprietà, bellezza della lingua e altre fisime da retori» e «furono con intelligenza, però, con discrezione, come del resto voleva il Leopardi, aperti verso il nuovo almeno in senso letterario e culturale» (1967: 17).

Rispetto ai primi studi sulla ricezione rondesca, Boni compie un passo avanti, individuando non quale Leopardi i rondisti abbiano tradito, ma quale abbiano voluto valorizzare. Proprio qui sta il punto: spostare lo sguardo sulla ricezione verso la comprensione delle motivazioni che portarono certe epoche, certi intellettuali o certe riviste a interpretare in una maniera o nell'altra un autore. Per fare ciò bisogna operare una rivoluzione copernicana, allontanare lo sguardo dall'autore recepito ed entrare nella prospettiva del soggetto ricevente ${ }^{1}$. Scrive BONI nel suo saggio:

Direi pertanto che l'interpretazione e l'accoglimento della lezione leopardiana da parte dei rondisti ebbe [...] dei limiti anche gravi, fu parziale ma non fu affatto un tradimento come sostiene il Bo. Anzi, della lezione leopardiana, i rondisti misero in luce aspetti nuovi e comunque trascurati fino ad allora e in momenti in cui erano particolarmente utili e necessari. E ciò fu fatto con calmo e fermo entusiasmo e con i rondisti il Leopardi divenne veramente una voce viva nella cultura italiana contemporanea. (1967:22-23)

Reinquadrare la ricezione in una prospettiva sincronica, tenendo conto dell'epoca di cui si parla: questo è il punto di partenza per capire il Leopardi de «La Ronda» e delle altre riviste che prenderemo in esame. Sulla questione della ricezione rondesca di Leopardi torna SOLMI nel 1972 con il saggio Leopardi e la Ronda, in cui si pone la domanda: «Ma il riproporsi dell'esempio di un grande poeta a tanta distanza di tempo, non rappresenta forse, sempre, un fraintendimento?» (1974 : 128), superando l'impasse su cui si erano chiusi molti dei suoi predecessori. Non si tratta di capire quanto l'originale lezione leopardiana sia stata attesa o disattesa, dal momento che in ogni epoca ci troviamo all'interno di un punto di vista che potrebbe risultare fuorviante. Quello che importa è capire quanto quella particolare ricezione sia stata importante per gli scrittori e i critici dell'epoca e quale sia stato il fine di una particolare lettura. Per questa ragione nel presente studio si prescinderà dal contestare le interpretazioni di Leopardi date dalle varie riviste, opinioni che possono risultare ai nostri occhi giuste o sbagliate, ma la cui correttezza non rientra nel fine che ci si prefissa: quello di registrare come Leopardi sia stato recepito ed utilizzato in alcune significative riviste italiane degli anni Venti e Trenta del secolo passato.

\section{La riscoperta de «La Ronda»}

Pare obbligatorio iniziare con qualche riferimento alla ricezione leopardiana de «La Ronda». I rondisti creano una sorta di mito di Leopardi, innalzandolo a loro modello. Basterebbe come prova di tale affermazione il suo inserimento nella rubrica "I convitati di pietra", attraverso la quale i redattori rendono omaggio a

\footnotetext{
${ }^{1}$ Si propone di allargare al campo della ricezione le teorie formulate negli studi sulla traduzione da studiosi come Toury ed Even-Zohar, che mettono al centro della questione il contesto di arrivo (Cfr. NERGAARD, 1995).
} 
personaggi della tradizione letteraria che considerano come maestri. Nel corso dei suoi tre anni di vita, «La Ronda» dedica a Leopardi più di trecento pagine considerando nel calcolo solo i suoi testi e gli articoli a lui dedicati ed escludendo i riferimenti in articoli di carattere generale o di altro argomento. Il contributo de «La Ronda» alla scoperta novecentesca di Leopardi non è meramente quantitativo, ma la sua rilevanza sta nell'impatto che ebbe su un'intera generazione di critici e scrittori che attraverso le sue pagine scoprirono Leopardi. Ce ne dà una testimonianza SOLMI, che riferendosi alla lettura cardarelliana di Leopardi scrive:

È doveroso da parte nostra riconoscere che La favola breve di Leopardi esercitò un notevole fascino sulle nostre giovinezze. Cardarelli, per noi, veniva a riattualizzare energicamente il Leopardi delle Operette accostandolo ai prestigiosi esemplari del decadentismo europeo, ai poemetti in prosa di Baudelaire e di Mallarmé, e in un modo, alla fin dei conti, non ingiustificato. (1974: 133)

Per dirla con BONI, l'azione dei rondisti «costrinse, direi quasi, l'uomo di cultura della prima metà del nostro secolo ad avvicinarsi al Leopardi» (1967 : 30). Viene da chiedersi perché gli scrittori de «La Ronda» scelsero Leopardi, cosa li attirò nella sua figura e nella sua opera. Un tassello interessante per far luce sulla questione è un articolo uscito sulla rivista nell'aprile del 1920, in cui i rondisti dichiarano che il panorama delle lettere italiane risulta minacciato da quattro tendenze nocive: dannunzianesimo, pascolismo, futurismo e letteratura frivola. Per questo motivo hanno deciso di riallacciarsi al momento in cui la grande tradizione italiana sembra essersi interrotta, ossia a Manzoni e a Leopardi. Ciò che i rondisti vedono in Leopardi, e che li avvicina a lui, è l'equilibrio tra classicismo e modernità. Dopo Leopardi sarebbe cominciato il declino delle lettere italiane, causato secondo i rondisti dall'avvio del Risorgimento, che da una parte ha spostato l'attenzione degli intellettuali sulla politica e dall'altra ha interrotto i collegamenti con la grande rete intellettuale europea ${ }^{2}$. Il cosmopolitismo culturale di Leopardi è un'altra delle caratteristiche che colpisce i rondisti, che dedicano un intero capitolo del numero sullo Zibaldone a estratti sulle letterature straniere moderne. I rondisti, spesso accusati di nazionalismo e chiusura verso le letterature straniere, furono invece molto ricettivi verso ciò che accadeva al di fuori dei confini nazionali e apprezzarono in Leopardi questa stessa disposizione (LANGELLA, 2002).

Altri elementi leopardiani accolti dai rondisti sono la rivalutazione del Cinquecento, l'idea che l'arte nasca dall'immaginazione ma che per darle forma sia necessario un intenso lavoro e un certo gusto per l'ironia. Ciò che li attirò fu poi lo stile, in particolare quello delle Operette Morali, che grazie alla forza delle loro immagini diventano il modello di prosa dei rondisti (PULCE, 2000). «La Ronda» privilegiò il Leopardi prosatore e minore: ciò era senz'altro una risposta all'interpretazione crociana dei Canti come momento più alto dell'opera

\footnotetext{
${ }^{2}$ Una simile visione del Risorgimento può essere rintracciata in quello che De Sanctis scriveva su Leopardi: «Beati i tempi, quando, non essendo ancora fatta l'Italia, il maggior pensiero degl'Italiani era disputare intorno a un libro di filosofia o di poesia! Tempi sentimentali, meravigliosamente disposti a comprendere Faust, Manfredi, Amleto, Leopardi! [...] Ma quel mondo poetico non esiste più: cadde flagellato a sangue dall'ironia di Heine; cadde quando quella trista generazione di esuli che cercavano una patria si sentì soddisfatta, anzi oltrepassata, nelle sue speranze» (LONARDI, 1974 : 96).
} 
leopardiana, ma si confaceva anche alle posizioni letterarie della rivista ${ }^{3}$. Una rivalutazione rivendicata con sprezzo dallo stesso Cardarelli, che in un articolo apparso nel settembre 1920 scrive:

È poca gloria ma incontestabile che il primo a parlare in Italia, in questi ultimi anni, delle Operette e a disegnare questa parabola dell'opera leopardiana, sia stato io. Senza di che le mummie del dottor Ruysch non so quando si sarebbero risvegliate. (CARDARELLI, 1920)

All'interpretazione cardarelliana delle Operette morali molti avanzeranno critiche. Tra di essi, Solmi scriverà che secondo Cardarelli «il pessimismo leopardiano, le teorie morali e metafisiche espresse in quel libro costituirebbero soltanto qualcosa come un pretesto a puri incanti di stile, capaci di suscitare, attraverso colorite mitologie, misteriosi panorami cosmici» (1974: 131). Se volessimo ribaltare in positivo questo giudizio, diremmo che la lettura di Cardarelli tende a rivelare l'elemento poetico di questi testi e quell'attenzione allo stile che è propria della poetica de «La Ronda». Parlando delle Operette Morali, CARDARELLI tornerà a esaltare l'aspetto cosmopolita dell'opera di Leopardi, essendo questo «l'unico libro di pretto sapore europeo che sia stato scritto in Italia nel primo Ottocento» (1962 : 940-41). Bacchelli, dal canto suo, individua nelle Operette di Leopardi un esempio perfetto di poesia in prosa, inserendo il poeta nel discorso tutto personale sul rapporto tra prosa e poesia e in quello rondesco della morte della poesia in versi.

L'attenzione alla prosa di Leopardi determina anche l'interesse verso il Leopardi pensatore e filosofo, evidente ad esempio nel numero dedicato allo Zibaldone. Nel 1930 CARDARELLI scriverà, similmente a quanto aveva scritto per le Operette Morali: «Dovevamo venire noi de la "Ronda" a ricordare agl'italiani l'esistenza dello Zibaldone» (1962: 515). Per quanto riguarda lo Zibaldone ci troviamo in questi anni di fronte a una prima ricezione, giacché la sua pubblicazione risale ad appena vent'anni prima. Sul Leopardi minore torna anche Riccardo Bacchelli in un lungo saggio di due puntate sui Paralipomeni della Batracomiomachia che esce su «La Ronda» tra giugno e luglio del 1920, in cui invita a una lettura non solo politica ma anche poetica del poemetto leopardiano. La riscoperta del Leopardi minore determina una rimessa in gioco del poeta nella sua ricezione primonovecentesca. Il lavoro de «La Ronda» è in questo senso il punto di partenza dello sforzo intrapreso poi anche da altre riviste.

\section{2. «La Fiera Letteraria» e gli studi leopardiani}

In un trafiletto comparso il 28 febbraio del 1926 sulla prima pagina de «La Fiera Letteraria», Sisifo - pseudonimo di Ugo Ojetti - risponde stizzito all'invito del francese Edmond Jaloux agli italiani affinché riconoscano finalmente la grandezza

\footnotetext{
${ }^{3}$ Per il tono sprezzante con cui nella rivista si parla di Croce si veda la recensione di Aurelio Emilio Saffi al saggio su Baudelaire di Croce apparso su «La Critica» («La Ronda», I, 2, maggio 1919), o anche la recensione del suo saggio su Goethe di Marcello Cora («La Ronda», III, 1/2, gennaio/febbraio 1921), l'articolo di Ferrero Storia e storici nella critica di Benedetto Croce («La Ronda», III, 10, ottobre 1921) e Contraddizioni e incompetenza di un filosofo («La Ronda», IV, 3/4, marzo/aprile 1922).
} 
di Leopardi. Stizzito non perché non consideri Leopardi un grande scrittore, ma perché si additerebbe agli intellettuali italiani contemporanei la colpa di sottovalutare il poeta:

Quanto a Leopardi, siamo tutti orecchi per udire dalla bocca di Edmond Jaloux che cosa dobbiamo fare per onorarlo. Mai credo dal 1837 abbiamo tanto studiato, letto, ristampato, e anche imitato prosa e poesia di Giacomo Leopardi quanto oggi. Ma, se Edmond Jaloux non lo sa, questo, caro Cardarelli, caro de Robertis, caro Piccoli, è meno che niente. (SisIFO, 1926)

I toni sono smorzati nel numero successivo da Nino Frank, che scende in campo in difesa di Jaloux e della propria rivista "Augustea», che aveva pubblicato il giudizio del francese. Fatto sta che - forse a seguito dell'accusa di Jaloux, forse per un caso - dall'anno successivo «La Fiera letteraria» inizierà a interessarsi con costanza di Leopardi, pubblicando tra il 1927 e il 1928 undici interventi su di lui. Il primo di essi esce nella rubrica "Specola della scienza" e si inserisce nel contesto di un referendum che, prendendo come spunto la descrizione della caduta della luna contenuta nel sogno di Alceta, chiede ai lettori un'opinione su quanto grande, a loro parere, Leopardi vedesse la luna. Attraverso lo spunto leopardiano si invitano i lettori a riflessioni di tipo astronomico, ottico, ma anche letterario. Gli interessi leopardiani della rivista rientrano però ben presto all'interno dell'ambito della letteratura in senso stretto. Il 1927 è un anno leopardiano, se stiamo a quanto dice Flora nell'incipit del suo articolo Fortuna di Leopardi:

\footnotetext{
Non credo si sia mai tanto parlato di Leopardi quanto in quest'anno. Le occasioni più varie hanno offerto gli spunti: o fosse l'apparizione della chiara Elegia di Soffici, o la polemica sull'endecasillabo, o lo studio che il Citanna vien pubblicando ne La Critica, o l'edizione dei Canti, curata da Moroncini. (FLORA, 1927)
}

La breve rassegna prosegue con i saggi leopardiani annunciati in uscita, soffermandosi in particolare su quello di Zottoli per Laterza. È un chiaro esempio di come le riviste possano essere un utile strumento per capire la ricezione di un autore in un determinato momento. Dopo aver fatto il punto sullo stato degli studi leopardiani, il critico cerca di capire come Leopardi sia interpretato dagli scrittori contemporanei:

Non direi che uno scrittore così divulgato nelle scuole, e che è perfino mandato a memoria dagli studenti, sia oggi più vicino all'intimo spirito letterario della nostra stagione, di quanto fosse alla generazione giovane che lo vide morire [...] Anche oggi, come allora, accanto ai più convinti fedeli, vi sono scrittori pei quali la figura di Leopardi è soltanto letteraria: sicché li lascia immuni: ed altri vi sono per i quali è una figura nemica, che si ammira ma non si ama, e che infine bisogna scacciare dal proprio spirito. (FLORA, 1927)

Flora sente il pericolo che Leopardi diventi un mito, un nome tanto famoso quanto enigmatico, e che la sua opera resti in fondo poco conosciuta. Ciò che secondo Flora ostacola nell'avvicinarsi a Leopardi, rendendolo quasi un nemico, è l'alta percezione del dolore della vita e la profondità dei suoi «pensieri di varia filosofia». Il critico ne loda il canto dispiegato e la capacità di far diventare «musica novissima» il linguaggio «trito di antica letteratura»e alla fine propone una 
soluzione: per ristabilire la giusta grandezza della letteratura ottocentesca e di Leopardi bisogna prima accettare la scala gerarchica teorizzata da Alfieri, che vedeva in Dante, Petrarca, Ariosto e Tasso i quattro grandi della letteratura italiana. Il critico propone di inserire Leopardi in un discorso sulla letteratura italiana storiografico e canonizzante, per favorime la comprensione da parte dei contemporanei. Il discorso di Flora dimostra che a questa altezza cronologica si impone una questione di ordine storiografico sulla ricezione di Leopardi. Seppur ancora legato alle categorie di classico e romantico, di cui Leopardi costituirebbe la mediazione, Flora sente il problema di collocarlo nel canone dei grandi poeti italiani. Leopardi viene inserito nella tradizione italiana, della quale era figlio e all'interno della quale operò egli stesso delle scelte, prima tra tutte la rivalutazione del modello cinquecentesco ${ }^{4}$.

«La Fiera Letteraria» registra in due anni di interesse leopardiano l'intenso lavoro che andavano svolgendo critici e filologi. Vengono segnalati i lavori sui Canti di Migliorini, Moroncini e De Robertis, la pubblicazione delle lettere inedite di Fanny Targioni Tozzetti e Antonio Ranieri su «Nuova Antologia» e altri studi sulle carte napoletane. Tra di essi spicca il curioso articoletto Versi incomprensibili di Leopardi, in cui si commenta uno studio di Porena su alcuni versi apparentemente senza senso ritrovati tra le carte di Leopardi, che sarebbero in realtà un promemoria in codice inventato dal poeta per la stesura della Storia di un'anima scritta da Giulio Rivalta. «La Fiera Letteraria» mostra un'attenzione verso Leopardi che possiamo definire filologica, finalizzata alla scoperta di nuovi testi o di aspetti poco noti dell'opera e della biografia dell'autore. La rivista è luogo di dibattito e divulgazione, aliena da un orientamento forte come quello de «La Ronda» e per questo più obiettiva, nel senso che si astiene dal proporre una propria visione del poeta, preferendo registrare il punto sullo stato degli studi e le novità editoriali. In questo senso possiamo considerare il ruolo de «La Fiera letteraria» più neutro rispetto a quello dei rondisti e testimonianza di un dibattito che iniziava ad essere vivo tra gli intellettuali italiani.

\section{Leopardi strapaesano}

Se «La Fiera letteraria» è una testimonianza neutra del dibattito su Leopardi, completamente differente è il caso de «Il Selvaggio» di Mino Maccari, in cui tra la fine degli anni Venti e i primi anni Trenta si nota un forte interesse per Leopardi. «Il Selvaggio» nasce nel 1924 come rivista allineata con le politiche del nascente regime; ma già nel 1926, sotto la direzione di Maccari, inizia ad allontanarsi dalla linea ufficiale del partito, ormai distante dagli ideali rivoluzionari e antiborghesi che

\footnotetext{
${ }^{4}$ L'interpretazione di Flora dovette avere una certa risonanza, se in uno libro sulla ricezione leopardiana del 1968 Ferdinando GIANNESI scrive: «E chiudiamo con queste pagine di Francesco Flora: l'esempio - fino ad oggi - della critica leopardiana più complesso ed equilibrato. Qui la varietà degli elementi considerati è ridotta ad armonia di giudizio, e ogni tendenza polemica, ogni gusto precipitoso di scoperta, vengon superati in una visione totale e simultanea del problema. Di qui, partirà la nuova critica leopardiana di domani» (1968: 188). Il saggio di Flora di cui parla Giannesi è Poetica del Leopardi, ma vi si nota la stessa tendenza dell'articolo de «La Fiera Letteraria».
} 
animano la rivista ${ }^{5}$. «Il Selvaggio» iniziò allora da una parte a dettare una propria linea che, seppur interna al fascismo, si opponeva alle correnti dominanti creando malumori nel partito, dall'altra a dedicarsi più assiduamente all'arte, alla letteratura e alla satira. È in questo contesto che va inserito l'interesse per Leopardi. Scriverà Maccari molti anni dopo ricordando l'esperienza giovanile: «A me piacevano Montaigne, Voltaire, Leopardi e Schopenhauer, gli spiriti liberi». Per i selvaggi Leopardi è simbolo di ribellione e forza d'animo, contrapposto alle frivolezze di scrittori come Salgari o De Amicis. Uno degli eventi scatenanti della ripresa di Leopardi è un articolo apparso sulla rivista «Raduno», periodico del sindacato fascista degli scrittori, in cui si leggeva: «Io affermo che nel bilancio storico dell'Italia, della nuova Italia, Emilio Salgari conta molto più che Giacomo Leopardi» (RAGGHIANTI: 1994: 69-70). Nel numero del 30 gennaio 1928 i selvaggi rispondono nella maniera che gli è più congeniale, con una vignetta satirica in cui si vedono i redattori di «Raduno» che innalzano un monumento a Carolina Invernizio, popolare autrice di romanzi d'appendice, con la seguente didascalia:

Grazie al «Raduno» ai veri valori del genio italiano si rende finalmente giustizia. Dopo Salgari, Carolina Invernizio avrà il suo posto che si merita. Bistolfi ne prepara la statua, e, alla chetichella, Mondadori ne va stampando l'«opera omnia». Ma a Strapaese si dorme con un occhio solo.

Leopardi letto dai selvaggi finisce per essere un Leopardi funzionale agli scopi e alla linea politica e letteraria della rivista. La prassi de «Il Selvaggio», simile a quella de «La Ronda», è quella di estrarre lunghe citazioni dagli scritti di Leopardi. Attraverso questa tecnica di decontestualizzazione e grazie all'abbondanza di materiali derivanti dallo Zibaldone e dall'epistolario leopardiano, i redattori della rivista possono individuare i passi leopardiani funzionali a sostenere le loro idee. Il primo estratto risale al maggio del 1928 ed è intitolato Leopardi alla XVI Biennale. Si tratta di un passo dallo Zibaldone del 27 luglio 1923, in cui Leopardi esalta la semplicità e la chiarezza nell'arte, auspicando la scomparsa dell'autore in favore di una rappresentazione il più possibile vicina al vero. Un passo che ben si addice alle idee del movimento di Strapaese, di cui «Il Selvaggio» è espressione. Si tenta di far passare l'idea di un Leopardi strapaesano, come appare anche nel trafiletto Leopardi contro la Torre d'Avorio, pubblicato su «Il Selvaggio» il 15 febbraio 1929:

Andando dietro ai versi e alle frivolezze (io parlo qui generalmente) noi facciamo espresso servizio ai nostri tiranni; perché riduciamo a un gioco o a un passatempo la letteratura; dalla quale sola potrebbe aver solo principio la rigenerazione della nostra patria.

La ricerca di un Leopardi anticipatore delle poetiche strapaesane diventa esplicita nel numero del 30 giugno 1929 in un articolo che, sotto il titolo Il sostegno a Strapaese, cita alcuni passi leopardiani. Per difendersi dagli attacchi di Stracittà, i redattori della rivista decidono «che ci si valga [...] soprattutto dell'autorità e della sapienza dei grandi di tutti i tempi; i quali si sono posti a disposizione del Selvaggio

\footnotetext{
${ }^{5}$ Vittoria CORTI identifica l'ideologia di Maccari con quella della parte sovversiva del fascismo, quella che «aveva creduto che si sarebbe davvero arrivati alla rivoluzione e alla giustizia sociale, e stava aspettando ancora, con impazienza, che questo avvenisse» (1994: 12).
} 
per sostenerne le campagne e le ragioni». Tra di essi c'è il «marchigiano Leopardi; amico nostro, e non della ventura». Se per i rondisti Leopardi era un modello di stile, l'ultimo grande classico della letteratura italiana, per i selvaggi è un anticipatore e un sostenitore delle loro idee. I passi leopardiani sono citati sotto titoli funzionali alla loro interpretazione tendenziosa: uno di essi ad esempio viene chiamato Novecentismo, vecchio vizio, in polemica con i redattori della rivista «900». I passi estratti dall'opera leopardiana si concentrano su due temi: da una parte la contrarietà verso la prassi sempre più diffusa tra $\mathrm{i}$ «poetuzzi italiani» di imitare le mode letterarie straniere, che svilirebbe l'originalità e il genio italiano, dall'altra il primato degli antichi, dei greci e dei latini, e la diretta discendenza da essi della letteratura italiana.

Altro punto della poetica leopardiana che piacque molto ai selvaggi fu la critica della ragione, interpretata dai redattori della rivista come critica al cerebralismo tipico della linea novecentista e modernista. Nel numero del $1^{\circ}$ aprile 1933 viene pubblicata una citazione leopardiana su questo tema, mentre il 15 giugno dello stesso anno sarà la volta di un passo sull'immaginazione, intesa come contraltare di una ragione troppo pervasiva. Attraverso l'immaginazione gli antichi hanno saputo raggiungere l'eternità e la gloria, che ai moderni sono precluse dall'uso della ragione. Un discorso che gli strapaesani traducono in una svalutazione del presente e in un conservatorismo rivoluzionario che guarda alla tradizione come punto di partenza per uno stravolgimento del presente. Tuttavia, in Leopardi non c'è nessuna possibilità di tornare indietro: l'avvento della ragione e la perdita delle illusioni non sono un processo reversibile, ciò che è perduto è perduto per sempre, come Leopardi spiega in altri passi della sua opera. I selvaggi lo ignorano, e anzi il 15 dicembre del 1933 citano un passo in cui Leopardi esordisce così:

Se i Principi risuscitassero le illusioni, dessero vita e spirito ai popoli e sentimento di se stessi, rianimassero con qualche sostanza, con qualche realtà gli errori e le immaginazioni costitutrici e fondamentali delle nazioni e delle società, se ci restituissero una patria, se il trionfo, se i concorsi pubblici, i giuochi, le feste patriottiche, gli onori renduti al merito ed ai servizi prestati alla patria tornassero in usanza; tutte le nazioni certamente acquisterebbero o piuttosto risorgerebbero a vita e diverrebbero grandi e forti e formidabili.

Un'ipotesi che in Leopardi assume il sapore dell'adynaton, ma che i selvaggi prendono come proposito, desiderio auspicabile e realizzabile. La parabola leopardiana de «Il Selvaggio» culmina nel novembre del 1934 con la pubblicazione integrale del Discorso sui costumi degli italiani. Molto significative sono ancora una volta le parole premesse dai redattori, in cui viene confermata la lettura in positivo di Leopardi: le parole del poeta sullo stato dell'Italia sono ancora attuali e la loro lettura è consigliabile «a quanti [...] si adoperano alla rinascita della nostra nazione». La tendenza è quella di applicare, attraverso l'elemento paratestuale e la selezione delle citazioni, una pars construens al discorso leopardiano, proiettando le constatazioni filosofiche e storiche di Leopardi in una struttura di pensiero progressista. Ancora una volta i selvaggi rivendicano per Leopardi il ruolo di rappresentante delle loro idee: 
Lo scritto del Leopardi costituisce un'ottima guida, e, per quel che ci riguarda, può, in genere, esprimere come noi non sapremmo, il fondamento della nostra polemica, e preservarla dalle degenerazioni e dagli immiserimenti a cui non per colpa nostra ma per la grande malafede e furberia di tanta gente, è tratta ad ogni piè sospinto. Ma se noi possiamo esser tenuti in iscacco dall'abilità e sofisticheria e opportunismo degli avversari, valga Leopardi a schiacciarli. È anche nel loro interesse farsi mettere a cuccia da una così alta autorità.

Il poeta è usato come scudo contro gli attacchi degli avversari e in tale funzione è implicita la sua strumentalizzazione. «Il Selvaggio» offre una lettura di Leopardi ideologicamente orientata, prediligendo il Leopardi civile, fautore di un'arte genuina, fondata sulla natura e sugli antichi, e portatore di un temperamento ribelle e irrequieto in cui i redattori si riconoscono. Completamente assente il Leopardi lirico e quello materialista delle Operette morali. Ciò che è presente è lo Zibaldone, ma la selezione dei passi mira allo scopo unico di portare Leopardi sulle posizioni sostenute dalla rivista.

\section{Il centenario leopardiano}

Il 1937 è l'anno del centenario della morte di Leopardi: molte sono le celebrazioni e anche le riviste non perdono occasione per dedicargli attenzione. Su alcuni almanacchi letterari di quell'anno escono articoli che sottolineano la ricorrenza. L'«Almanacco dei Visacci» pubblica un articolo in cui Raffaello Franchi accosta l'opera di Leopardi a quella di Giotto, di cui quell'anno ricorreva un altro centenario. Tra le considerazioni leopardiane, particolarmente significativa risulta la raccomandazione ai lettori di «leggere le pagine piane e formidabili dei Pensieri leopardiani, quelle delle sue Operette morali» poiché «solamente così essi si renderanno degni di accostarsi, con limpida mente, con cuore limpido, alle liriche» (FRANCHI, 1937). La visione di Franchi presuppone ancora il primato della poesia, ma sottolinea come la prosa sia strumento indispensabile per accostarsi al pensiero del poeta e capire fino in fondo la sua poesia.

Di impostazione più tradizionale è l'articolo pubblicato sull' "Almanacco della donna italiana» da Ettore Allodoli, che oscilla tra obiettività del dato biografico, qualche acuta notazione e una certa tendenza a presentare Leopardi come un poeta conservatore, civile e nazionalista. Il critico ammette, a differenza di quanto facevano i selvaggi, il rifiuto di Leopardi delle «illusioni di progresso e di miglioramento scientifico e umano» e individua il nucleo centrale del pensiero leopardiano nel «problema per lui solo predominante dell'infelicità umana» (ALLODOLI, 1937), ammettendo il ruolo di Leopardi come maestro di modernità e inserendolo tra i grandi della letteratura italiana. Allodoli predilige gli aspetti vicini alla lettura che il regime fascista avrebbe potuto dare del poeta. E così viene descritto con ammirazione il padre Monaldo, «fieramente attaccato ai principi legittimisti e cattolici, rafforzatisi tenacemente in lui dopo le invasioni francesi, e i soprusi, le violenze, le ruberie d'ogni specie, fatte in nome della libertà e dell'uguaglianza». Un'attitudine che il giovane Leopardi avrebbe ereditato:

Alla caduta del regime francese, nel 1815, Giacomo salutò con gioia il ritorno del legittimismo, e scriveva nella quiete della biblioteca paterna un saggio politico esortante gl'italiani ad unirsi perché fosse reso impossibile il ritorno del disordine e delle rivoluzioni. 
Fatto che potremmo anche considerare vero, se non fosse che Allodoli non ritiene necessario precisare i successivi sviluppi del pensiero leopardiano e l'allontanamento dalle idee del padre. Al Leopardi legittimista si affianca il Leopardi nazionalista, «poeta civile d'Italia» con le prime canzoni. L'articolo presenta il primo Leopardi come il Leopardi migliore, questa fase «illumina l'intima coscienza di questo poi appassionato negatore e distruttore di tutto», come se l'evoluzione del pensiero leopardiano fosse la corruzione di un originario pensiero conservatore. Seppur molto tra le righe, si capisce che la motivazione di questo deperimento del pensiero è da cercare nelle disgrazie del poeta, secondo una sperimentata tendenza a motivare il pensiero attraverso la vita di cui lo stesso Leopardi a suo tempo già si lamentava. Allodoli tuttavia non nasconde una sincera ammirazione per l'autore e sottolinea persino la componente ironica e sarcastica della sua opera. Un sarcasmo anch'esso figlio dell'infelicità, ma che avrebbe dato frutti da non trascurare, come i Paralipomeni della Batracomiomachia, di cui Allodoli auspica la riscoperta e nei quali vede però solo una critica ai progressisti.

Se gli almanacchi per loro stessa natura pongono particolare attenzione ai centenari, anche le altre riviste non passarono sotto silenzio la ricorrenza leopardiana. Ad interessarsi al poeta sono ad esempio «La Riforma Letteraria», «Meridiano di Roma» e «L'Orto», ma sarà «Il Frontespizio» la rivista più attiva sul fronte leopardiano nel 1937. A Leopardi i redattori de «Il Frontespizio» dedicano l'intero numero di settembre. Anche qui è presente la tendenza a leggere il Leopardi che più si confà all'ideologia della testata. Così «Il Frontespizio», rivista di orientamento cattolico, sceglie come epigrafe per il suo numero leopardiano la seguente citazione:

O la immaginazione tornerà in vigore, e le illusioni riprenderanno corpo e sostanza in una vita energica e mobile, e la vita tornerà ad essere cosa viva e non morta, e la grandezza e la bellezza delle cose torneranno a parere una sostanza, e la religione riacquisterà il suo credito; o questo mondo diventerà un serraglio di disperati, e forse anche un deserto.

Già nel 1935, su «Il Frontespizio» era apparso un passo di una lettera a Giordani come esempio del purismo portato avanti dalla rivista. Il numero dedicato a Leopardi si apre con un testo di Giovanni Papini dal titolo Leggenda argentea di Giacomo Leopardi poeta e martire, che racconta la vita del poeta seguendo il modello della Legenda Aurea di Jacopo da Varazze. La lettura di questo testo rivela un certo compiacimento eroicomico e un tono quasi parodico. Gli interventi del numero leopardiano assumono un particolare spessore, se consideriamo che tra $\mathrm{i}$ loro autori troviamo alcuni degli intellettuali più importanti dell'epoca, tra cui Carlo Bo, Mario Luzi e Carlo Betocchi. Il fascicolo risulta fondamentale per capire la ricezione di Leopardi da parte del mondo cattolico e dell'ermetismo fiorentino. Ci limiteremo qui a commentare il contributo di Betocchi, Leopardi e noi, in cui si traccia l'eredità di Leopardi nel panorama contemporaneo:

Se l'uomo ha bisogno, e questo bisogno ha sentito e sente più che mai nell'incremento assiduo della indagine scientifica e della speculazione filosofica, di una testimonianza di assoluta, 
inderogabile fedeltà a se stesso, questa testimonianza gli è data da Giacomo Leopardi (BETOCCHI, 1937).

Per i cattolici Leopardi riesce a trasmettere il senso della difficoltà del percorso che porta, attraverso il pensiero, all'accettazione della fede o al suo rifiuto. Leopardi è per Betocchi il campione della riflessione, esempio di una discesa faticosa all'interno dell'animo; e aggiunge, riferendosi ai cattolici, che «Leopardi non è uno che li tradisce; è uno che non mentisce, né si fa ingannare sulla nostra condizione». Leopardi, ateo e materialista, diventa per Betocchi una guida per chi sente il bisogno di ricercare la fede e il suo canto è un «canto dell'uomo», prima ancora che della natura o del dolore. Ancora una volta il poeta risulta trasportato all'interno dell'orizzonte di chi lo legge e lo interpreta.

Il centenario dovette avere un valore catalizzatore, dal momento che negli anni successivi Leopardi continuò a essere presente in maniera massiccia nelle riviste italiane. La presenza leopardiana è evidente sia in riviste indipendenti dalle politiche del regime, come «Letteratura» e "Campo di Marte», che in riviste allineate come «Primato» e «Maestrale». Le riviste testimoniano, attraverso le recensioni, il moltiplicarsi degli studi su Leopardi anche nell'editoria classica. Il lavoro svolto dalle riviste sembra aver dato i suoi frutti nel riproporre la questione Leopardi all'attenzione dei critici. Il dibattito si concentra intorno alla rivalutazione delle opere minori e dei testi in prosa di Leopardi, che come abbiamo visto si configura come reazione all'interpretazione crociana, ma anche come volontà di inserire il poeta nel canone della tradizione italiana e di farlo proprio da parte dei vari gruppi intellettuali dell'epoca. Queste due tendenze dimostrano la nuova importanza che Leopardi viene ad assumere in questo primo scorcio di Novecento e che sarà alla base della sua fortuna e delle varie influenze sugli scrittori italiani nel corso del secolo.

\section{BIBLIOGRAFIA}

Allodoli, Ettore (1937), Il centenario di Leopardi, Almanacco della donna italiana, XVIII.

BetocCHI Carlo (1937), Leopardi e noi, Il Frontespizio, IX, 9.

BONI Massimiliano (1967), Leopardi e La Ronda, Bologna, Edizioni Italiane Moderne.

CARDARELli Vincenzo (1920), Dico a te, nuora, La Ronda, settembre 1920.

CARDARElli Vincenzo (1962), Opere Complete, Milano, Mondadori, 1962.

CORTI Vittoria, Rosai e Maccari al tempo del Selvaggio fiorentino, Firenze, Giorgi \& Gambi Editori, 1994.

FLORA Francesco (1927), Fortuna di Leopardi, La Fiera Letteraria, III, 28, 17 luglio 1927.

FrANCHI, Franchi (1937), 1937. Anno di Leopardi e Giotto, "Almanacco dei Visacci», I, 1.

GIANNESI Ferdinando (1967), Giacomo Leopardi nel tempo, Milano, La Goliardica. 
LANGELla Giuseppe (2002), Passaporto per La Ronda, in RizzAnTE Massimo e GUBERT Carla (eds.), Le riviste dell'Europa letteraria, Trento, Editrice Università degli Studi di Trento, pp. 95-116.

LONARDI Gilberto (1974), Leopardismo. Tre saggi sugli usi di Leopardi dall'Otto al Novecento, Firenze, Sansoni.

NERGAARD Siri (1995), Teorie contemporanee della traduzione, Milano, Bompiani.

PULCE Graziella (2000), Gli scrittori della «Ronda» di fronte alle «Operette morali», in BELLUCCI Novella e CORTELLESSA Andrea (eds.) «Quel libro senza uguali». Le "Operette morali» e il Novecento italiano, Roma, Bulzoni, pp. 163-83.

RagGhianti Carlo Ludovico (1994), Il Selvaggio di Mino Maccari, Vicenza, Neri Pozza.

Sisifo, OJeTti Ugo (1926), Leopardi, La Fiera Letteraria, II, 9, 28 febbraio 1926.

Solmi Sergio (1974), Leopardi e La Ronda, in Leopardi e il Novecento, Atti del III Convegno Internazionale di Studi Leopardiani (Recanati 2-5 ottobre 1972), Firenze, Olschki. 